\title{
JUNCTION PARAMETER EXTRACTION FOR ELECTRONIC DEVICE CHARACTERIZATION
}

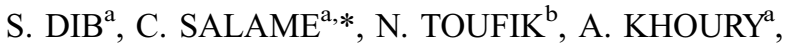 \\ F. PÉLANCHON ${ }^{\mathrm{b}}$ and P. MIALHE ${ }^{\mathrm{b}}$ \\ ${ }^{a}$ Laboratoire LPSE, Département de Physique, Université Libanaise-Faculté de Sciences II, \\ BP 90656 Jdeidet, Liban; ${ }^{\mathrm{b}}$ LP2A, Université de Perpignan, 52 Avenue de Villeneuve, \\ 66860 Perpignan Cedex, France
}

(Received 22 October 2002; In final form 17 November 2002)

\begin{abstract}
A new method for the extraction of junction parameters from a description of the current-voltage characteristic is developed. A simulation is performed and a high accuracy is obtained for the determination of the single exponential model parameters. The method is easy to implement in a control process for device characterization. An application, achieved to observe the degradation of the emitter-base junction of a bipolar transistor during an aging experiment, shows that the evolutions of the single exponential model parameters versus time introduce a means for degradation quantification.
\end{abstract}

Keywords: Junction; Parameters; Extraction; Modeling; Quality

\section{INTRODUCTION}

The $I-V$ characteristic of a $p-n$ silicon junction can be described [1] by the implicit equation:

$$
I=\frac{V-R_{S} I}{R_{\mathrm{sh}}}+I_{0}\left[\exp \left(\frac{V-R_{S} I}{A V_{T}}\right)-1\right]
$$

known as the single exponential model.

$V_{T}=q / k T$ is the thermal voltage. Parameters $A, R_{s}, R_{\mathrm{sh}}, I_{0}$ are determined so that Eq. (1) gives a good description of the experimental characteristics. The series $\left(R_{S}\right)$ and shunt $\left(R_{\mathrm{sh}}\right)$ resistances take into account power losses and leakage currents: their values have been connected to carrier concentration and were dependent on the device technological structure. Several theories [2-5] relating the value of the ideality factor, $A$, to carrier recombination via different traps have been published and optimization of junction performances has been performed. These parameters [6-8] are of interest for quality control since they are related to internal properties of the components. A method has been developed [9] to qualify microelectronics devices; they have been used to quantify radiation-induced damage [10] or electrical stress-induced degradation $[11,12]$.

* Corresponding author. E-mail: salame@ieee.org

ISSN 0882-7516 print; ISSN 1563-5031 online (C) 2004 Taylor \& Francis Ltd

DOI: $10.1080 / 1042015031000073805$ 
This work presents a new method to extract the junction parameters $A, R_{s}, R_{\mathrm{sh}}, I_{0}$, related to the one exponential model, from the experimental $(I-V)$ characteristic. A least square fitting of the $I-V$ characteristic is performed, using the high voltage region of the curve. The influence of the start values for computing processes is considered and the accuracy of the method is discussed. A simulation is performed to compare the results with theoretical values of the parameters. The method is applied to characterize the base-emitter junction of transistors.

\section{MODELING OF THE METHOD}

The acquisition of a junction $I-V$ characteristic yields $n$ experimental values $(I(j), V(j))$, $1 \leq j \leq n$. The method consists of extracting the estimated values of the parameters $A, R_{s}$, $R_{\text {sh }}, I_{0}$ in order to fit these $n$ experimental values $(I(j), V(j))$ from Eq. (1).

\subsection{Determination of $\mathrm{A}$}

From Eq. (1), I expresses as:

$$
I=I_{1}+I_{2}
$$

where

$$
\begin{aligned}
& I_{1}=I_{0}\left[\exp \left(\frac{V-R_{S} I}{A V_{T}}\right)-1\right] \\
& I_{2}=\frac{V-R_{S} I}{R_{\mathrm{sh}}}
\end{aligned}
$$

For values of the operating potential $V$ greater than $0.5 \mathrm{~V}, 1$ is negligible compared to $\exp \left(\left(V-R_{S} I\right) /\left(A V_{T}\right)\right)$ (see Appendix 1) and Eq. (3) becomes:

$$
V-R_{S} I=A V_{T} \ln \left(\frac{I_{1}}{I_{0}}\right) .
$$

Introducing

$$
Y=V-R_{s} I_{2}
$$

Eq. (5) writes:

$$
Y=R_{S} I_{1}+A V_{T} \ln \left(\frac{I_{1}}{I_{0}}\right) .
$$

First values of $R_{\mathrm{sh}}$ and $R_{S}$ are determined (Sec. 1.2) then, from the $n$ experimental $(I, V)$ values, $I_{2}, I_{1}$ and $Y$ can be computed through Eqs. (4), (2) and (7), respectively: this process yields $n$ values of function $Y\left(I_{1}\right)$ as given by Eq. (7).

The method considers arbitrary values $A^{\prime}, R_{s}^{\prime}, I_{0}^{\prime}$ of the parameters $A, R_{s}, I_{0}$ and Eq. (7) leads to:

$$
Y^{\prime}=R_{s}^{\prime} I_{1}+A^{\prime} V_{T} \ln \left(\frac{I_{1}}{I_{0}^{\prime}}\right)
$$


For a same current value (that is for a same $I_{1}$ value), the difference $(\Delta Y)$ between $Y$ and $Y^{\prime}$ writes:

$$
\Delta Y=Y^{\prime}-Y=\left(R_{s}^{\prime}-R_{s}\right) I_{1}+V_{T}\left(A^{\prime}-A\right) \ln I_{1}+A V_{T} \ln \left(\frac{I_{0}}{I_{0}^{\prime}}\right) .
$$

This method is based on the observation that for $A^{\prime}=A, \Delta Y$ is a linear function of $I_{1}$ and Eq. (9) becomes:

$$
\Delta Y=\left(R_{s}^{\prime}-R_{s}\right) I_{1}+A V_{T} \ln \left(\frac{I_{0}}{I_{0}^{\prime}}\right) .
$$

The research of the value $A^{\prime}=A$ uses the least square method and the well-known linear correlation coefficient $R^{2}$. Practically, we use $\mathrm{LR}_{2}=-\ln \left(1-R^{2}\right)$ instead of $R^{2}$ to provide a better visualization of the fit (if $R^{2}=0.99$, then $\mathrm{LR}_{2}=4.6 ; R^{2}=0.999, \mathrm{LR}_{2}=6.9$; $R^{2}=0.9999, \mathrm{LR}_{2}=9.21$ ).

\subsection{Determination of Parameters $I_{0}, R_{s}, R_{\text {sh }}$}

The determination of the ordinate " $b$ " at the origin [Eq. (10)] of the line $\Delta Y\left(I_{1}\right)$ yields:

$$
I_{0}=I_{0}^{\prime} \exp \left(\frac{b}{A V_{T}}\right)
$$

and the slope " $a$ " of the line $\Delta Y\left(I_{1}\right)$ verifies: $a=R_{s}^{\prime}-R_{s}$.

These calculations suppose initial values for series and shunt resistances. They may be obtained from the experimental $I(V)$ characteristics [13]. A first value of $R_{S}$ can also be easily computed using the above procedure with the approximation [14] of an infinite value of the shunt resistance. The shunt resistance $R_{\mathrm{sh}}$ can be measured from the reverse $I(V)$ characteristic [15]. The influence of these initial values on the accuracy of the method is discussed below.

\section{HOW TO TEST THE ACCURACY OF THE DETERMINATION}

Starting from an original (experimental) $I-V$ characteristic, the method successively extracts the (estimated) values of $R_{s}, R_{\mathrm{sh}}, A$ and $I_{0}$. Then, an estimated $I-V$ characteristic can be constructed from Eq. (1).

Beyond $V>0.5 \mathrm{~V}$, the $I-V$ curve is quasi vertical: a slight increase of $V$ yields a drastic enhancement of $I$. In order to consider this particularity and to improve the fitting, a wellknown function $Q[13,16]$ has been used in this study.

$$
Q=\left[\frac{1}{n} \sum_{i=1}^{n}\left(d_{i}\right)^{2}\right]^{1 / 2}
$$

where $d(k)$ is the distance between the points of the calculated characteristic and the experimental points, in relative units [13]. The parameter $Q$ appears as the root-mean-square of these $n$ distances. A perfect description of the experimental points would yield $Q=0$. A calculated value of $Q$ lower than $5 \times 10^{-3}$ corresponds to a good fit between the two characteristics.

\section{CORRECTNESS OF THE METHOD}

\subsection{Dependence of the Starting Values of $\boldsymbol{R}_{s}^{\prime}, I_{0}^{\prime}$}

The estimated values of the three parameters $A, R_{s}, I_{0}$ are derived using a choice of initial values $R_{s}^{\prime}$ and $I_{0}^{\prime}$. It is essential that this choice does not influence the final results. 
TABLE I Extreme Parameter Values Used for Computing the Ideal Characteristic.

\begin{tabular}{lcccc}
\hline & \multicolumn{4}{c}{ Parameters } \\
\cline { 2 - 5 } & $A$ & $R_{s}(\Omega)$ & $I_{0}(A)$ & $R_{s h}(\Omega)$ \\
\hline Minimal value & 1 & 0.01 & $10^{-16}$ & 50 \\
Maximal value & 2 & 5 & $10^{-6}$ & $\infty$ \\
\hline
\end{tabular}

The mathematical study of the different partial derivatives $\mathrm{d} A / \mathrm{d} R_{s}^{\prime}, \mathrm{d} A / \mathrm{d} I_{0}^{\prime}, \ldots$, is clearly impossible from a theoretical point of view. Thus, we have studied, in this paper, the variations of the "functions" $A, R_{s}, I_{0}$ versus the parameters (considered as variables), by the means of theoretical tables. Later, we have checked the correctness with experimental junction characteristics.

For instance, the variations of the extracted $A$ value versus the initial value $R_{s}^{\prime}$ have been studied with theoretical characteristics related to ideal junctions (Eq. (1)) corresponding to $R_{s}, I_{0}$ and $R_{\mathrm{sh}}$ values as referred to in Table I, $R_{s}^{\prime}$ varying in the range $[0.01,20] \Omega$.

Figures 1-2 display the respective variations of the extracted $A$ value versus initial values $R_{s}^{\prime}$ and $I_{0}^{\prime}$ and of extracted $I_{0}$ versus $R_{s}^{\prime}$ and $I_{0}^{\prime}$. The variation ranges are $[0.01,20] \Omega$ for $R_{s}^{\prime}$ and $\left[1.7 \times 10^{-16}, 1.7 \times 10^{-6}\right]$ A for $I_{0}^{\prime}$. It is noticeable that, except for the value $R_{s}^{\prime}=R_{s}$, the different observed surfaces are planar: this shows that the final results do not depend on the initial choices of $R_{s}^{\prime}$ and $I_{0}^{\prime}$.

\subsection{Accuracy of the Shunt Resistance Value and Influence on the Results}

The accuracy of the determination of the shunt resistance $R_{\mathrm{sh}}$ strongly depends on the accuracy of the measures of the current $I$ and the potential $V$. Figure 3 displays the $Q$ variations versus $R_{\mathrm{sh}}$, for an ideal characteristic $\left(A=1.4, R_{S}=2.5 \Omega, I_{0}=5 \times 10^{-9} \mathrm{~A}\right) . Q$ is all the lower as the $R_{\text {sh }}$ value is great, and $Q<5 \times 10^{-3}$ when $R_{\text {sh }}>100 \Omega$, which is a value largely exceeded with junctions in electronic devices.

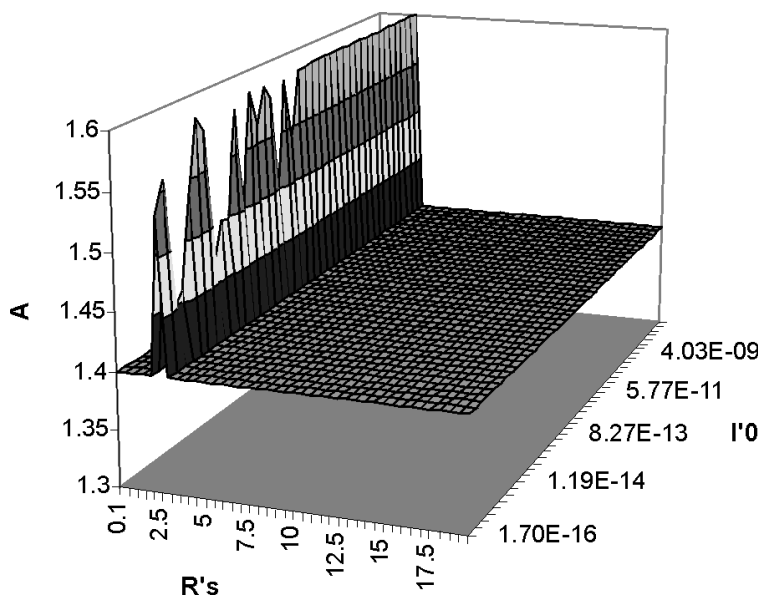

FIGURE 1 Variations of the extracted value of the ideality factor $A$ versus initial values $R_{s}^{\prime}$ and $I_{0}^{\prime}$ for an ideal characteristic $\left(A=1.4 ; R_{s}=2.5 \Omega ; R_{\mathrm{sh}}=5000 \Omega ; I_{0}=5 \times 10^{-9} \mathrm{~A}\right)$. The computed surface is planar except for $R_{s}^{\prime}=2.5 \Omega$. 


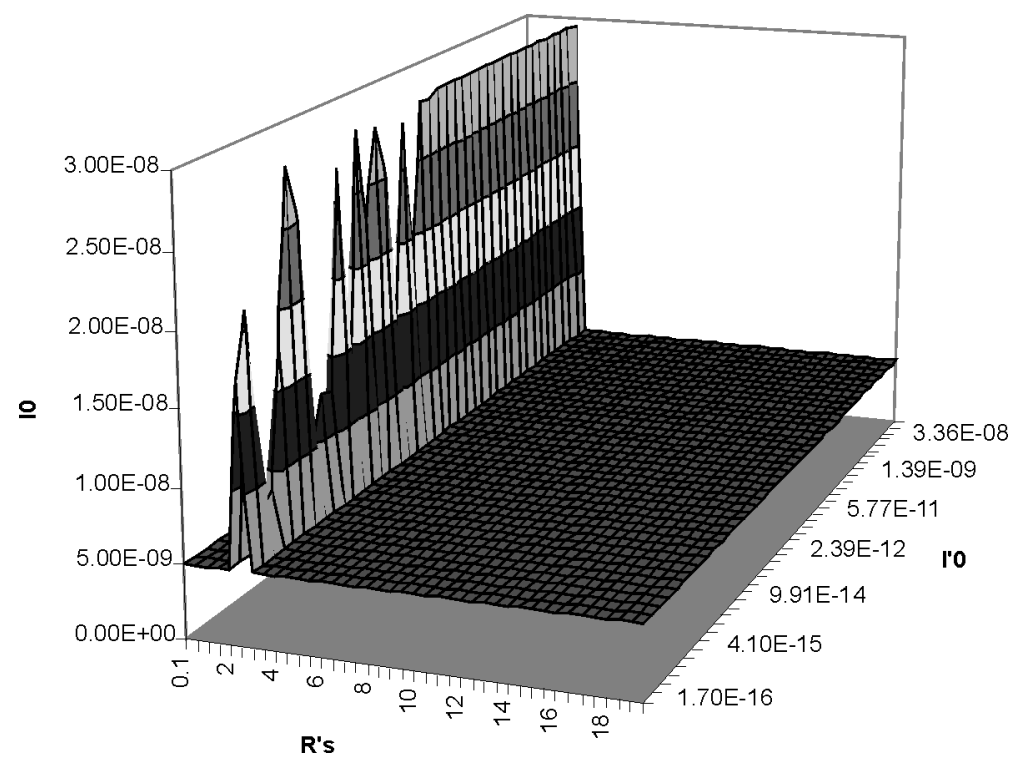

FIGURE 2 Variations of the extracted value of the ideality factor $I_{0}$ versus initial values $R_{s}^{\prime}$ and $I_{0}^{\prime}$ for an ideal characteristic $\left(A=1.4 ; R_{s}=2.5 \Omega ; R_{\mathrm{sh}}=5000 \Omega ; I_{0}=5 \times 10^{-9} \mathrm{~A}\right)$. The computed surface is planar except for $R_{s}^{\prime}=2.5 \Omega$.

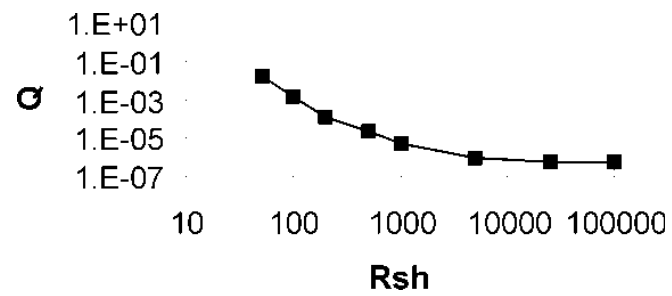

FIGURE $3 Q$ function versus $R_{\text {sh }}$ for an ideal characteristic $\left(A=1.4 ; R_{s}=2.5 \Omega ; I_{0}=5 \times 10^{-9} \mathrm{~A}\right)$.

\section{EXPERIMENT}

An electrical aging of npn bipolar transistors has been performed in order to investigate the gradual degradation in time of the emitter-base junction parameters. It is attributed [17] to hot carrier injection along the periphery of the junction in the space charge region of the emitter-base interface.

The devices used in this experiment were npn bipolar transistor, manufactured 2N2222A type. The electrical stressing was realized with a reverse bias, Vbe $=-8 \mathrm{~V}$, applied to the emitter-base junction with the collector open. The value, $60 \mathrm{~mA}$, of the reversed current biased the junction well into avalanche breakdown. At each step the junction current-voltage characteristic was measured and the junction parameters have been extracted.

Figure 4 shows the increase of the reverse diffusion-recombination current $I_{0}$ of the emitter-base junction with the stress time. This increase points out a degradation since it is related to recombination centres at the interface and in the space charge region of the junction. The variations of the series resistance $R_{s}$ with the stress time, Figure 5, shows that strong degradation of $R_{s}$ appears after a short stress time, then the degradation rate becomes smaller. 


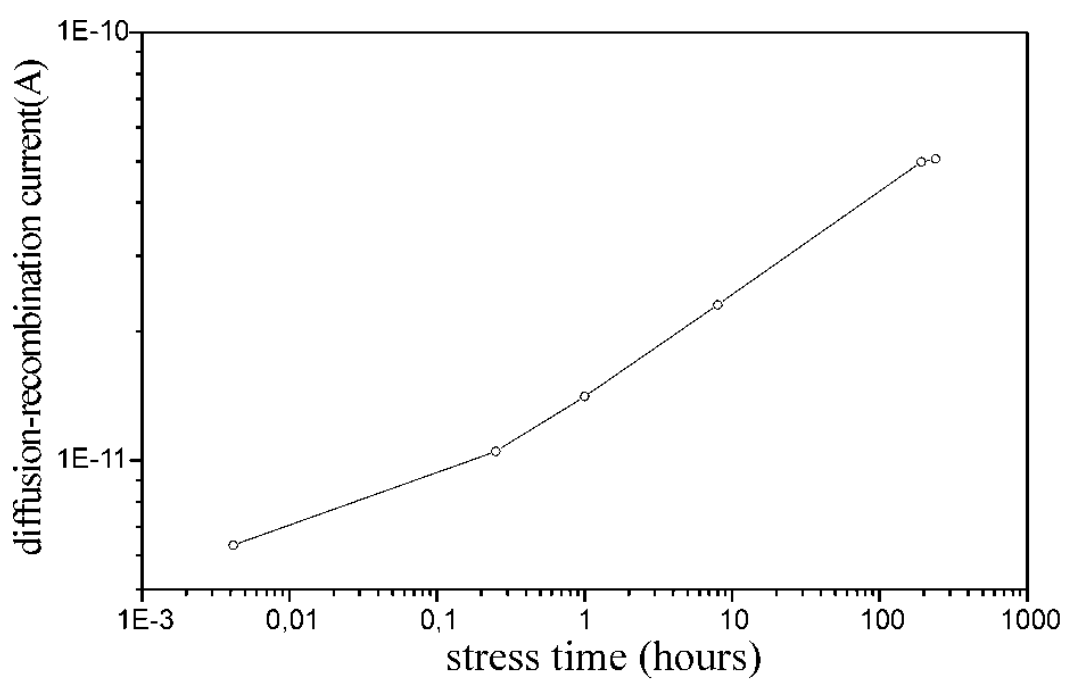

FIGURE 4 The extracted value of the reverse diffusion-recombination current versus the stress time for the emitter-base junction of a $2 \mathrm{~N} 2222 \mathrm{~A}$ transistor.

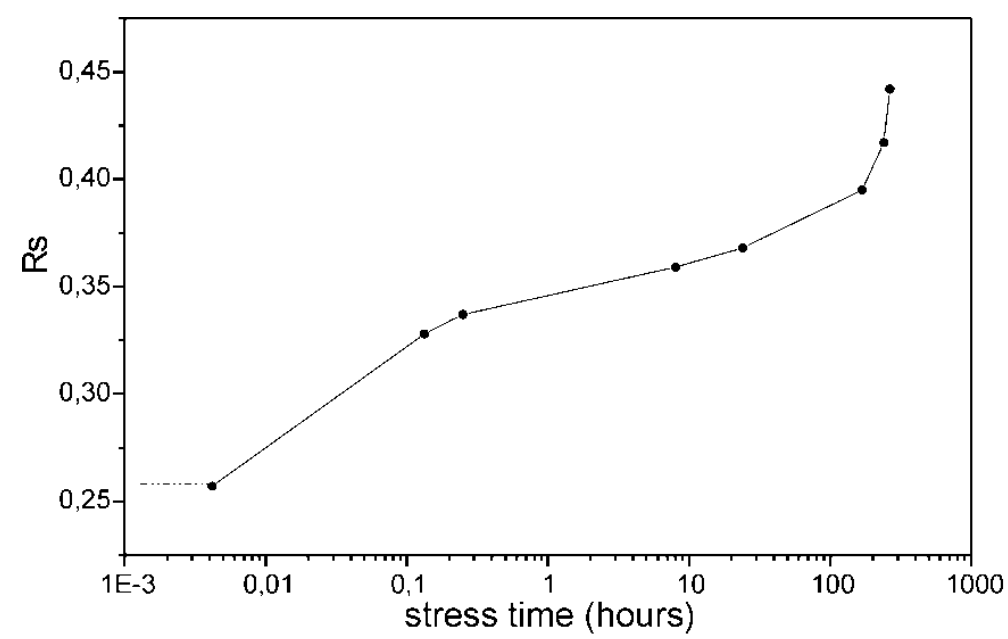

FIGURE 5 The series resistance $R_{s}$ of the emitter-base junction of a $2 \mathrm{~N} 2222 \mathrm{~A}$ transistor versus the stress time.

Series resistance and reverse diffusion-recombination current of the emitter-base junction appear strongly sensitive to the stress time.

\section{CONCLUSION}

A single exponential model has been used to describe the current-voltage characteristic of junctions in electronic devices. A new method has been developed to extract the parameters which are introduced and related to the device's physical properties. The accuracy of the method is shown to be independent of the starting values introduced in the computer process 
as soon as the shunt resistance is higher than $100 \Omega$. The method is applied to follow a device degradation obtained by an electrical stress and the evolution of the junction single exponential model parameters introduce a means for degradation quantification. The rapidity of the procedure and the simplicity of implanting the process in any program make it useful to quantify the quality of any junction.

\section{References}

[1] Sze, S. M. (1981). Physics of Semiconductors Devices, 2nd ed., Wiley-Interscience.

[2] Sah, C.-T., Noyce, R. N. and Shockley, W. (1957). Proc. IRE, 45, 1228.

[3] Pélanchon, F. and Mialhe, P. (1990). Solid-St Electron., 33, 47.

[4] Henry, C. H., Loyan, R. A. and Meritt, F. R. (1978). Appl. Phys., 49, 3530

[5] Pallares, J., Marsal, L. F., Correig, X., Calderer, J. and Alcubila, R. (1997). Solid-St Electron., $41,17$.

[6] Veissid, N., Bonnet, H. and Richter, H. (1995). Solid-St Electron., 38, 1937.

[7] Gottschalg, R., Rommel, M., Infield, D. G. and Kearney, M. J. (1999). Meas. Sci. Technol., 10, 796.

[8] del Cueto, J. A. (1999). Solar Energy Materials \& Solar Cells, 59, 393.

[9] de la Bardonnie, M., Toufik, N., Salamé, C., Dib, S. M., Mialhe, P., Hoffmann, A. and Charles, J.-P. (1999). Microelectro. Reliab., 39, 751.

[10] de la Bardonnie, M., Mahouad, A., Mialhe, P., Elmazria, O., Hoffmann, A., Lepley, B. and Charles, J.-P. (1995). IEEE Trans. on Nuclear Sc., 42, 1622.

[11] de la Bardonnie, M., Mialhe, P. and Charles, J.-P. (1998). J. of Phys. D: Appl. Phys., 31, 150.

[12] Doyle, B. S., Mistry, K. R. and Hung, C.-L. (1995). IEEE Trans. Elec. Dev., 42, 116.

[13] Charles, J. P., Mekkaoui-Alaoui, I., Bordure, G. and Mialhe, P. (1985). Solid-St Electron., 28, 807.

[14] Dib, S., de la Bardonnie, M., Khoury, A., Pélanchon, F. and Mialhe, P. (1999). Active and Passive Elect. Comp., 22, 157.

[15] Chakrabaty, K. and Singh, S. N. (1996). Solid-St Electron., 39, 577.

[16] Probabilité et Statistique, Cours et problèmes, Série Schaum, 1992, 271 and 274.

[17] Kosier, S. L., Wei, A., Schrimpf, R. D., Fleetwood, D. M., Delaus, M. D., Pease, R. L. and Combs, W. E. (1995). IEEE Trans. Elec. Dev., 42, 436.

\section{APPENDIX 1}

1 is negligible compared to $\exp \left(\left(V-R_{S} I\right) /\left(A V_{T}\right)\right)$ :

In any junction, $R_{S}$ is lower or of the order of a few $\Omega$. Then, when $V>0.5 \mathrm{~V}, R_{S} I$ is negligible compared to $V$ and $\exp \left(\left(V-R_{S} I\right) /\left(A V_{T}\right)\right)$ is practically equal $\exp \left(V /\left(A V_{T}\right)\right)$.

This value to $\exp \left(V /\left(A V_{T}\right)\right)$ is minimal when $A=2$ : it is greater than $\exp \left(0.5 /\left(2 V_{T}\right)\right)$ that is of the order of $15,000 \gg 1$. 

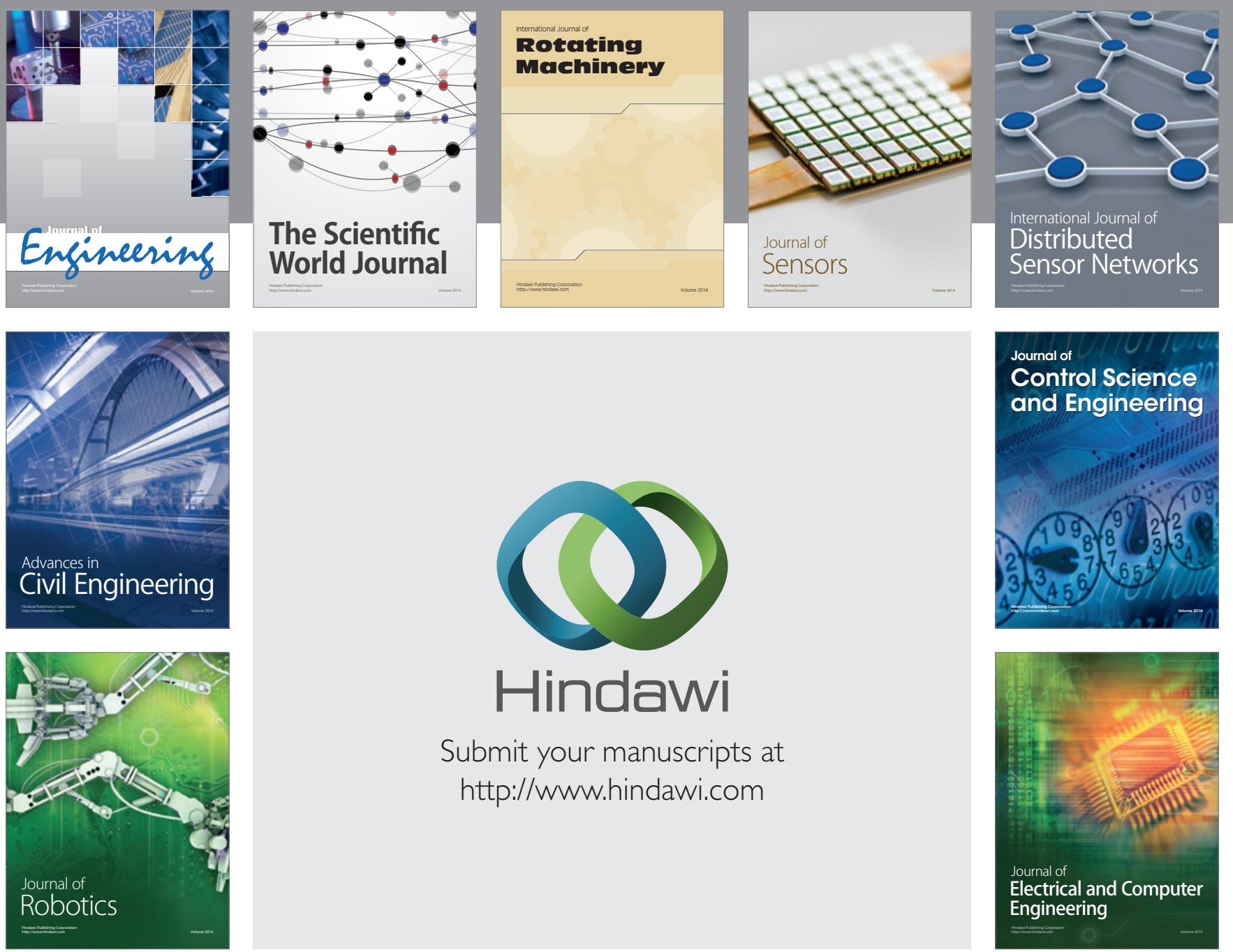

Submit your manuscripts at

http://www.hindawi.com
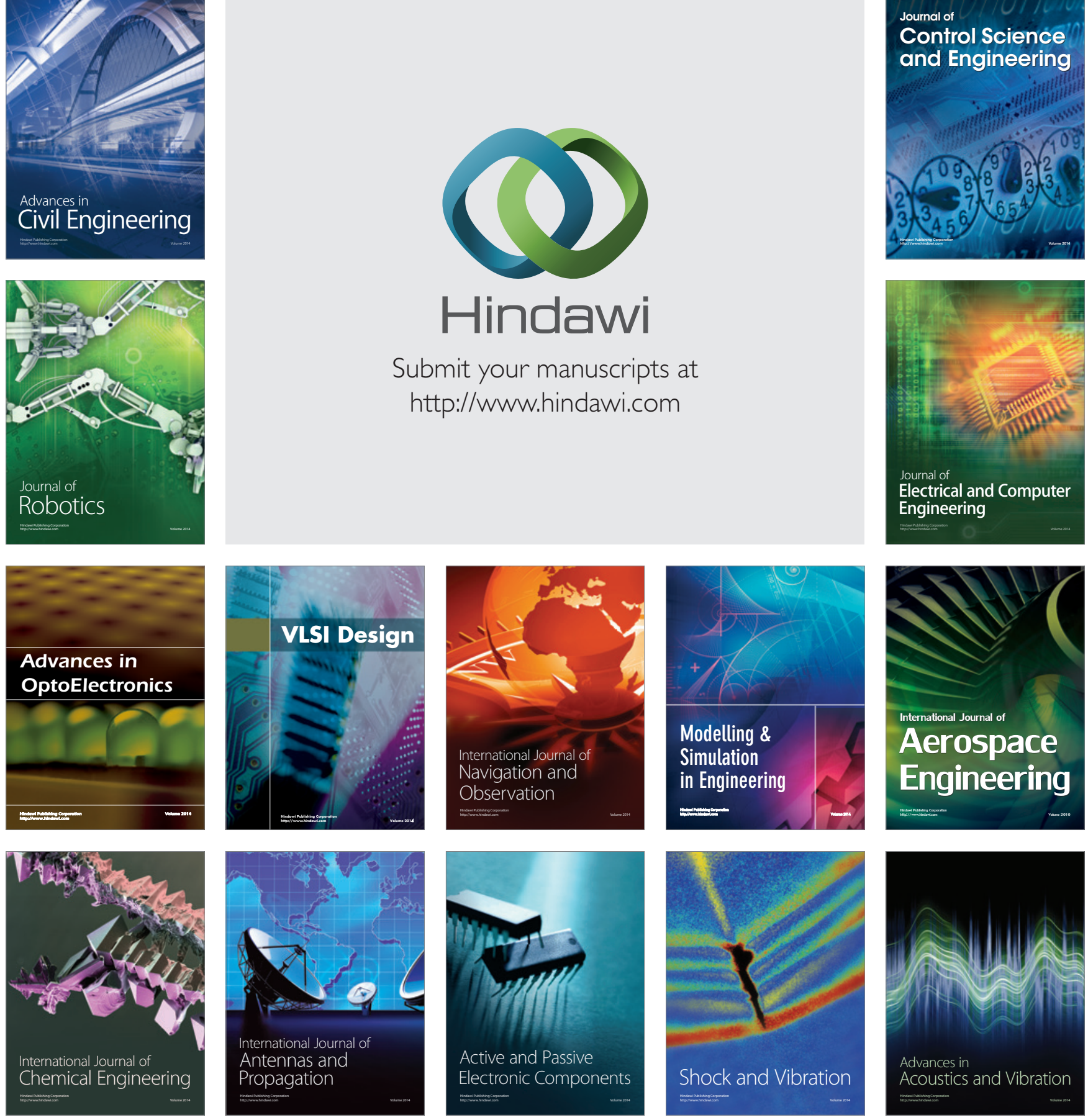